\title{
Quantized Mitochondrial Metabolism
}

\author{
Ioannis Rekkas ${ }^{1}$, Panagiotis Kordatzis ${ }^{2}$
}

\begin{abstract}
Mitochondrial biology is a rapidly growing sector, attracting the attention of many researchers. In particular, research on processes related to reactive oxygen species (ROS) and understanding of the risks associated with their production at mammalian mitochondria, has made significant progress in recent years. ROS production process has found to play a role in the development of diseases such as Alzheimer, Parkinson's, cancer, aging etc. Despite the fact that combating ROS has been the focus of many research groups, there has not been yet any proposal for a mechanism that could explain how mitochondrion produces free radicals. Such a mechanism would allow fighting ROS by limiting deregulated mitochondrial performance or by even restoring mitochondrial function, producing the proper amounts of water $\left(\mathrm{H}_{2} \mathrm{O}\right)$ and ATP.

This study proposes a model for the mitochondrial production of free radicals and suggests a mechanism to discover mitochondrial dysfunction.
\end{abstract}

Keywords: Abrikosov's quantum flux, Alzheimer, cancer, Parkinson's, ROS.

\section{Introduction}

The processes described and studied in the science of Physics could not be lacking in ability to describe processes involving biological systems. The effort made in this work is based precisely on this kind of cooperation: effects of Physics (Quantum Mechanics) applied in Biology, also helping to establish Quantum Biology. The phenomenon of Physics, which mainly appears in this work, is that of superconductivity. From the literature on superconductivity, references are used in this work in particular to Cooper Pairs [1], the Quantization of Magnetic Flux (namely the Abrikosov's vortex) [2], [3] and the effect of Josephson's junction [4]. The application of these effects to mitochondrial structure and functioning conditions, produces a model that describes mitochondrial function and subsequently ROS production.

\section{Mechanism of Superconducting Break}

\subsection{Localization.}

(Part 2.1 provides a general view of mitochondrion and determines the region that the proposed mechanism operates.)

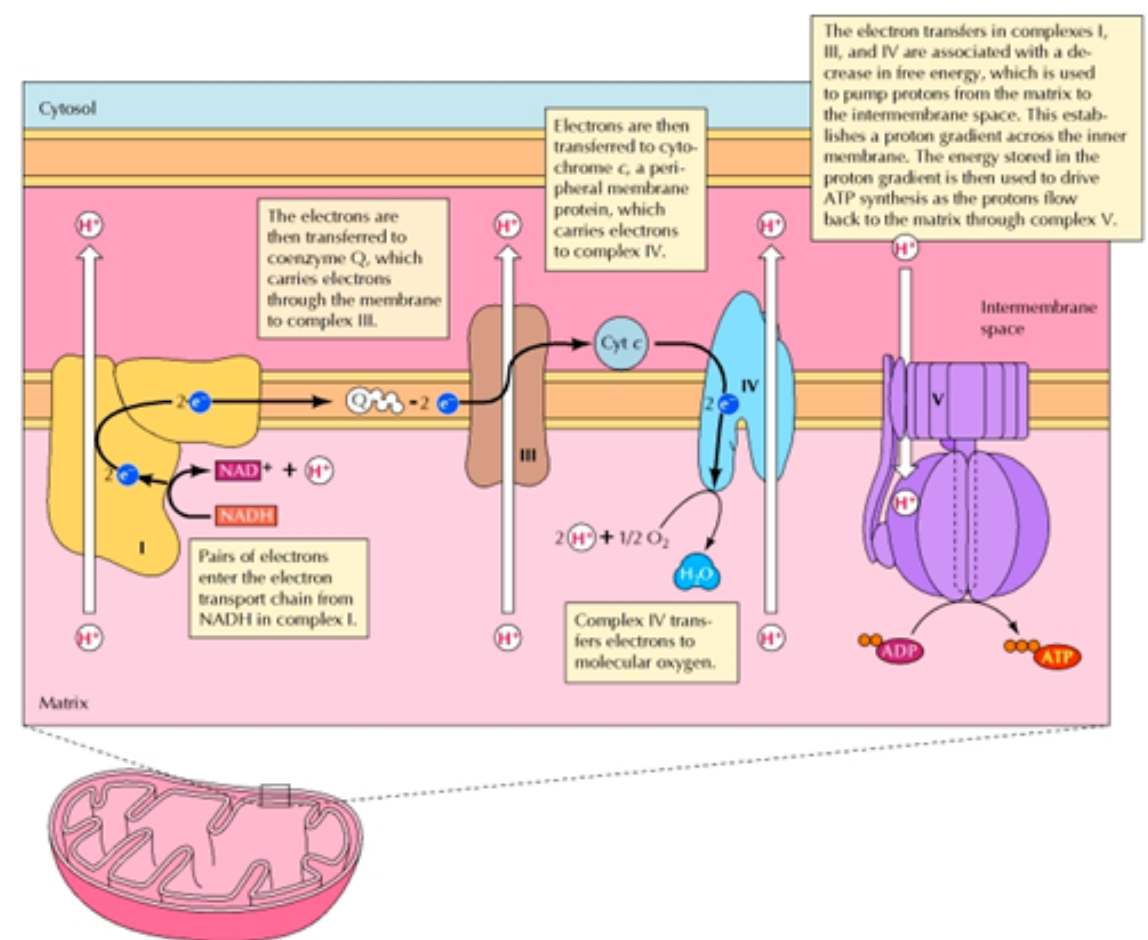

Figure 1: Mitochondrial schematic and the corner of complex IV (the blue exit). This corner is the area we focus on this work. 


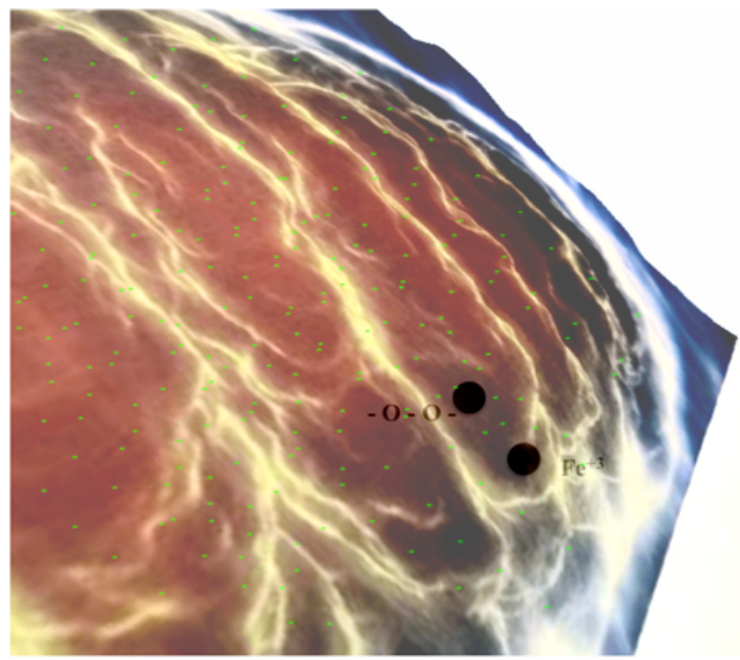

Figure 2: Description of how $\mathrm{Fe}^{+3}$ and - $\mathrm{O}-\mathrm{O}$ - are distributed by the exit of complex IV. There is also a depiction of negative charge located at the inside of the inner membrane. Charge is everywhere on the inner membrane and is distributed in a rather uniform way. For all figures following, charge is assumed there but is not shown.

Fig. 1 [5] shows the classic description of mitochondrion and focuses in the area just inside the inner membrane. This is the limited space in which the superconducting effect unfolds. Specifically the area of interest is where complex IV reaches the internal area of the membrane. The mechanism, hereinafter discussed, occurs at the corner where the electrons leave complex IV.Electrons coming from complex IV enter this particular space where potential difference is applied (electric field, Fig. 2). This potential is between the inside of the inner membrane and the outside. The potential is negative within the inner membrane and positive at the outside [6]. Therefore, electrons "swim" inside a closed space where applied electrical forces forbidding them to approach the walls. Due to the charged and closed inner surface, electrons are directed to the molecule of heme. Specifically, arrive as Cooper Pairs (CP) at the area where iron's atom (Fe) is bonded to two oxygen atoms -OO- (Fig. 2). This closed charged surface is considered as "electrical cage", namely Paul Trap [7]. The electrical cage effect plays a significant role in directing incoming electrons in the most accurate way possible.

\subsection{The superconducting "runaway"}

(Part 2.2 completes the description of the conditions in the area under examination and focuses specifically in the small area of interest.)

Fig. 3 shows the arrangement of iron $+3\left(\mathrm{Fe}^{+3}\right)$, two oxygen atoms $(-\mathrm{O}-\mathrm{O}-)$ and copper $+1\left(\mathrm{Cu}^{+1}\right)$ inside mitochondrion, with $\mathrm{Fe}^{+3}$ placed by the wall of the inner membrane. $\mathrm{Fe}^{+3}$ with the $-\mathrm{O}-\mathrm{O}-$ and $\mathrm{Cu}^{+1}$ form a natural superconducting surface at mitochondrion temperature.

Voltage difference between $\mathrm{Fe}^{+3}$ and $\mathrm{Cu}^{+1}$ favors movement of negative charge from $\mathrm{Cu}^{+1}$ to $\mathrm{Fe}^{+3}$ and the superconducting thin surface functions as a corridor used to accelerate negative charges towards $\mathrm{Fe}^{+3}$. On this corridor, two problems are hosted:

1) Active $\mathrm{Fe}^{+3}$ must be converted into less active $\mathrm{Fe}^{+2}$.

2) -O-O- must be detached from metals and contribute to water production. The second problem is actually the way to detach -O-O-, because the mechanism used could have two possible outcomes: It may convert -O-O- into $\mathrm{O}_{2}$ and then - normally - participate to $\mathrm{H}_{2} \mathrm{O}$ synthesis, or it may convert -O-O- into ROS. If ROS is produced (which is a major problem by its self), another problem appears: $\mathrm{H}_{2} \mathrm{O}$ is not synthesized, which leads to decreased amount of ATP.

To summarize the scenery: $\mathrm{Fe}^{+3},-\mathrm{O}-\mathrm{O}-$ and $\mathrm{Cu}^{+1}$ form a natural superconducting surface, inside a natural cage of negative charge. Incoming electrons from IV complex, coupled as CPs, cannot go anywhere else but towards $\mathrm{Cu}^{+1}$, attracted by its charge.

\subsection{Quantum shooting with Cooper bullets}

(In part 2.3 the proposed mechanism is explained.)

When carriers bring an electron pair inside, $\mathrm{Cu}^{+1}$ attracts the pair to the entry point of superconductor. This way, the CP acquires kinetic energy and the superconductor is activated (Fig. 4). The electron pair is accelerated towards -O-O-. CP is assisted by Paul trap effect to achieve the directionality required, to penetrate the potential barrier of -O-O- and break bonds between oxygen atoms and ions of metals. The result of this process, if successful, would be threefold. With two CPs: 


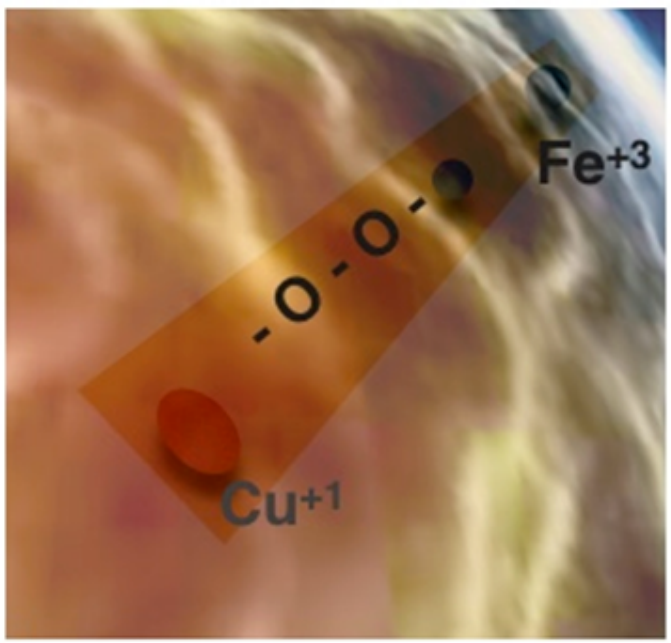

Figure 3: Superconducting plateau.

1. $\mathrm{Cu}^{+1}$ would convert into $\mathrm{Cu}^{+2}$.

2. O-O- accompanied by CPs would produce molecular oxygen, which would no longer be retained by ions of metals. Thus, would be deposited at the point where, along with four hydrogen ions, would form water (Fig. $5)$.

3. Water composition:

$4 \mathrm{H}^{+}+4 \mathrm{e}^{-}+\mathrm{O}_{2} \rightarrow 2 \mathrm{H}_{2} \mathrm{O}$

$\mathrm{Fe}^{+3}$ would convert into $\mathrm{Fe}^{+2}$ and would be removed by suitable carriers.

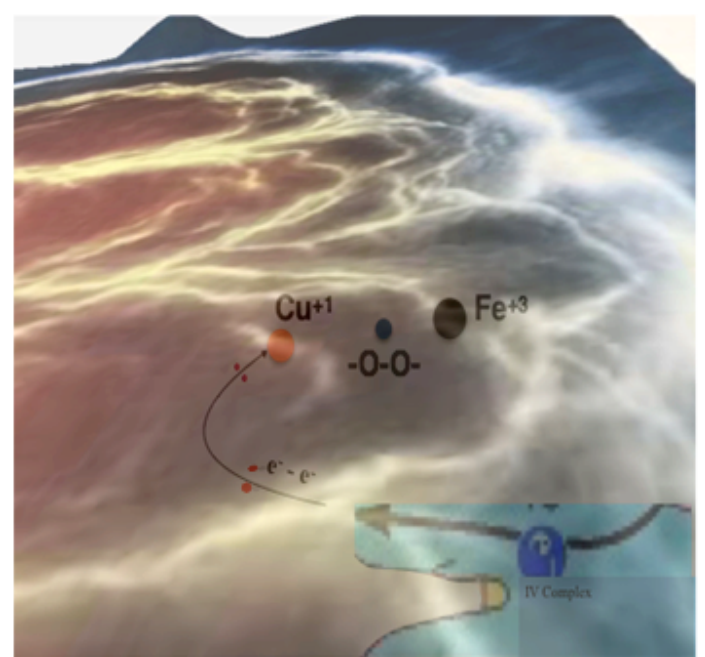

Figure 4: Incoming electron pair, attracted by $\mathrm{Cu}^{+1}$ and a second pair following.

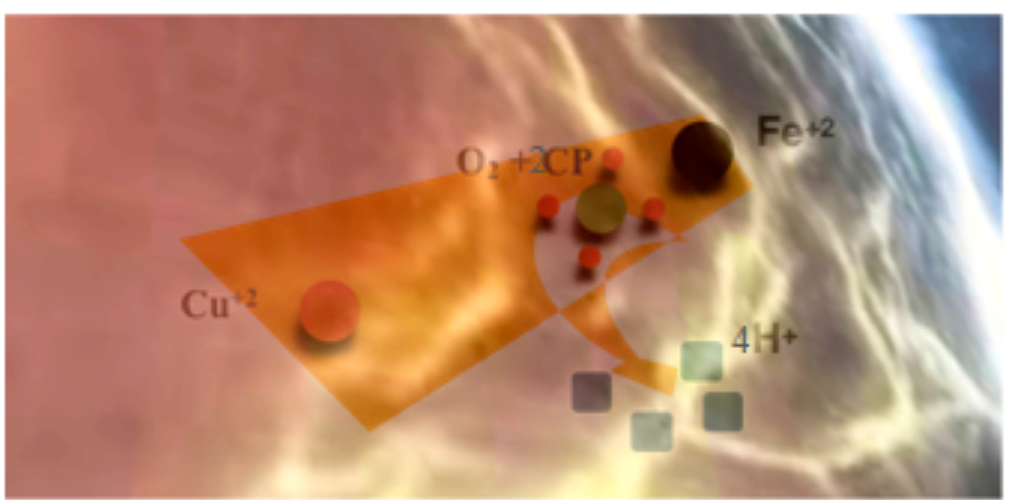

Figure 5: The arrow shows the point of the "triple meeting". $4 \mathrm{H}^{+}, \mathrm{O}_{2}$ and $2 \mathrm{CP}$ coming together to form water. Superconductor is now OFF! 
But, how $\mathrm{Fe}^{+3}$ and $\mathrm{Cu}^{+1}$ change their oxidation states? Perhaps the model proposed here could be used to describe the mechanism for redox reaction as well. In more detail, as described above the mechanism uses two CPs. The first CP comes in, approaches $\mathrm{Cu}^{+1}$ because is attracted by its charge, gains kinetic energy because is accelerated by voltage difference and repels common electron pair of $\mathrm{Cu}^{+1}-\mathrm{O}-\mathrm{O}-$ bond, towards $-\mathrm{O}-\mathrm{O}-$, converting $\mathrm{Cu}^{+1}$ into $\mathrm{Cu}^{+2}$. This also clears the way for next $\mathrm{CP}$. Second $\mathrm{CP}$ approaches $\mathrm{Cu}^{+2}$ attracted by its charge and again is accelerated due to voltage. Voltage for second CP is not the same as for the first one, because is accelerated between $\mathrm{Fe}^{+3}$ and $\mathrm{Cu}^{+2}$ and not between $\mathrm{Fe}^{+3}$ and $\mathrm{Cu}^{+1}$. However, is accelerated for longer distance, because there is no bond between $-\mathrm{O}-\mathrm{O}-$ and $\mathrm{Cu}^{+1}$, which counteracts the reduced voltage. Second pair reaches the bond $\mathrm{Fe}^{+3}-\mathrm{O}-\mathrm{O}-$ and their common electron pair is repelled towards $\mathrm{Fe}^{+3}$ converting it into $\mathrm{Fe}^{+2}$. The -O-O- along with the common electrons gained, is converted into molecular oxygen $\left(\mathrm{O}_{2}\right)$ and also binds all two CPs. This O2-CP mix will bond with four $\mathrm{H}^{+}$and produce water. The superconductor is deactivated (voltage between $\mathrm{Fe}^{+2}$ and $\mathrm{Cu}^{+2}$ is zero now).

In the ideal situation, the superconductor remains active for two CPs, then becomes inactive and is reactivated when new amounts $\mathrm{Fe}^{+3}$ and -O-O- are found. This superconducting procedure could not get better timing, as the amount of energy needed to break the bonds $\mathrm{Fe}^{+3}-\mathrm{O}-\mathrm{O}-\mathrm{Cu}^{+1}$ is large, and mitochondrion already expended much energy to transport the electrons at the appropriate point (exit of complex IV).

To summarize the proposed function: $\mathrm{CP}$, under the natural conditions inside mitochondrion, is attracted to $\mathrm{Cu}^{+1}$, as soon as it comes out of complex IV. When it comes at the point where $\mathrm{Cu}^{+1}$ is located, CP is exposed to voltage between $\mathrm{Fe}^{+3}$ and $\mathrm{Cu}^{+1}$ and is accelerated towards $\mathrm{Fe}^{+3}$. On its way it hits the -O-O-. The process is repeated with a second $\mathrm{CP}$. If everything is done correctly, the result is: conversion of $\mathrm{Fe}^{+3}$ to $\mathrm{Fe}^{+2}$ and $\mathrm{Cu}^{+1}$ to $\mathrm{Cu}^{+2}$ and $\mathrm{O}_{2}$ is produced to participate, along with two CPs, to water production reaction. It is clear now that this procedure is like using cooper pairs as bullets to shoot oxygen bonds, which justifies the title.

\section{Mathematical study}

(Part 3 is the mathematical description of the proposed model. It is consisted of three main subparts, the first to calculate the natural frequency of CP's, the second to calculate the frequency variation and the third to produce values using the formulas already derived. Natural frequency is derived using two approaches and a third approach, leading to the same result, is explained.)

\subsection{Calculating the natural frequency $\left(f_{0}\right)$}

\subsubsection{First approach or "Abrikosov \& Faraday" method}

Faraday's law describes that, changes in magnetic flux $(\mathrm{d} \Phi)$, happening within time dt, produce voltage ( V). If each CP completes its run within time $\mathrm{T}_{0}\left(\mathrm{~T}_{0}\right.$ is then - by definition - the period of the activation / deactivation phenomenon), the change of magnetic flux is $\Delta \Phi$ and the corresponding voltage is $\mathrm{V}_{0}$ (voltage between $\mathrm{Fe}^{+3}$ and $\mathrm{Cu}^{+1}$ ), then Faraday's law can be written as:

$\mathrm{V}_{0}=\frac{\Delta \Phi}{\mathrm{T}_{0}}$

The natural frequency $f_{0}$ is related to period. (2) describes this relation:

$\mathrm{f}_{0}=\frac{1}{\mathrm{~T}_{0}}$

Combining (2) and (1), (3) is derived:

$\mathrm{V}_{0}=\Delta \Phi \cdot f_{0}(3)$

According to Abrikosov, each $\mathrm{CP}$ absorbs one quantum of magnetic flux:

$\Phi_{0}=\frac{\mathrm{h}}{2 \cdot \mathrm{e}}$

where $\mathrm{h}$ is Planck's constant and $\mathrm{e}$ the elementary charge. In equation (3) $\Delta \Phi$ is the magnetic flux corresponding to one CP, so $\Phi_{0}=\Delta \Phi$ and (3), (4) combined produce:

$\mathrm{V}_{0}=\frac{\mathrm{h}}{2 \cdot \mathrm{e}} \cdot \mathrm{f}_{0}$

(5) gives the value of $\mathrm{f}_{0}$ :

$\mathrm{f}_{0}=\frac{2 \cdot \mathrm{e} \cdot \mathrm{V}_{0}}{\mathrm{~h}}$ 


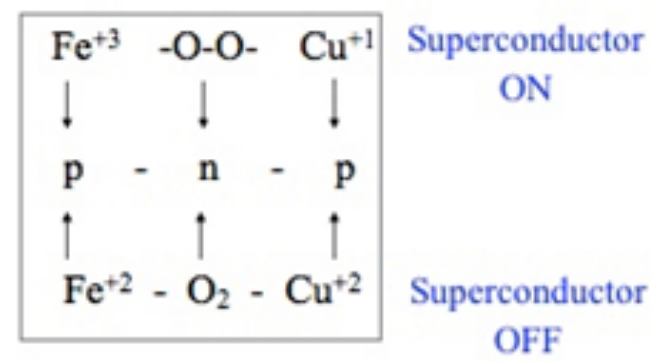

Figure 6: Correspondence to p-n-p semiconductor

\subsubsection{Second approach or "Josephson" method}

Study of Josephson junction with alternating current (ac) concludes that, whenever a CP penetrates $\mathrm{p}-\mathrm{n}-\mathrm{p}$ junction, a photon is emitted or absorbed. The photon's frequency is: $f_{0}=\frac{2 \cdot e \cdot V_{0}}{h}$ which is again equation (6). So, study as $\mathrm{p}-\mathrm{n}-\mathrm{p}$ junction that is targeted by $\mathrm{CP}$ (see analogy at Fig. 6), gives the same frequency. Also, the energy relationship $h \cdot f_{0}=2 \cdot e \cdot V_{0}$ for $C P$ could be used, to calculate $f_{0}$. This is a mere calculation that neither highlights theimportance of $\mathrm{f}_{0}, \mathrm{~V}_{0}$ nor allows the study of factors affecting the phenomenon. Nevertheless, it contributes to the completeness of studied hypothesis of superconductivity appearing when $\mathrm{CP}$ passes through the contact $\mathrm{Fe}^{+3}-\mathrm{O}-\mathrm{O}-\mathrm{Cu}^{+1}$.

\subsection{Doppler frequency variation ( $\Delta \mathrm{f}$ )}

For each $\mathrm{CP}$, kinetic energy has an "appropriate" range of values, which can cause coordination and finally wrest the oxygen from its ties. These limits are due to Doppler variation and can be determined by the thermal kinetic energy for each CP. Studying the pair as boson with two degrees of freedom (for more degrees of freedom the present method can easily expand), it is possible to equate kinetic energy of $\mathrm{CP}$ with thermal kinetic energy:

$\frac{1}{2} \cdot \mathrm{m}_{\mathrm{CP}} \cdot \overline{v^{2}}=\frac{3}{2} \cdot \mathrm{k}_{\mathrm{B}} \cdot \mathrm{T}(7)$

where $\mathrm{T}$ is the temperature, $\mathrm{k}_{\mathrm{B}}$ is the Boltzmann constant, $\mathrm{m}_{\mathrm{CP}}$ is CP's mass and $\overline{v^{2}}$ is the mean value of the squared velocities, for the couple of electrons. Solving (7) for $\overline{v^{2}},(8)$ is produced:

$$
\overline{v^{2}}=\frac{3 \cdot \mathrm{k}_{\mathrm{B}} \cdot \mathrm{T}}{\mathrm{m}_{\mathrm{CP}}}
$$

If $v_{\mathrm{e} 1}$ and $v_{\mathrm{e} 2}$ are the velocities for each electron of the pair, it is true that $v_{\mathrm{e} 1}=v_{\mathrm{e} 1}=v$, because electrons in CP move together - as a couple and $v_{\mathrm{e} 1}=v_{\mathrm{e} 1}=v$ is also the mean value of their motion. So $\overline{v^{2}}$ can be calculated as:

$\overline{v^{2}}=\frac{v_{\mathrm{e} 1}^{2}+v_{\mathrm{e} 2}^{2}}{2}=\frac{v^{2}+v^{2}}{2}=v^{2}$

(8) then becomes:

$$
v^{2}=\frac{3 \cdot \mathrm{k}_{\mathrm{B}} \cdot \mathrm{T}}{\mathrm{m}_{\mathrm{CP}}}
$$

and (10) can now be solved for $v$.

$v=\sqrt{\frac{3 \cdot \mathrm{k}_{\mathrm{B}} \cdot \mathrm{T}}{\mathrm{m}_{\mathrm{CP}}}}$

$v$ is needed in the relationship for Doppler variation, which is:

$\frac{\Delta \mathrm{f}}{\mathrm{f}_{0}}=\frac{\mathrm{v}}{\mathrm{c}}$

where $\mathrm{C}$ is the velocity of light in vacuum. Substituting (11) into (12) gives: 
$\Delta \mathrm{f}=\frac{\sqrt{\frac{3 \cdot \mathrm{k}_{\mathrm{B}} \cdot \mathrm{T}}{\mathrm{m}_{\mathrm{CP}}}}}{\mathrm{c}} \cdot \mathrm{f}_{0}$

By using (6), the fact that $\mathrm{m}_{\mathrm{CP}}=2 \cdot \mathrm{m}_{\mathrm{e}}$, where $\mathrm{m}_{\mathrm{e}}$ is the mass of each electron and by bringing everything under the root, (14) is produced:

$\Delta \mathrm{f}=\sqrt{\frac{6 \cdot \mathrm{k}_{\mathrm{B}} \cdot \mathrm{T} \cdot \mathrm{e}^{2} \cdot \mathrm{V}_{0}^{2}}{\mathrm{~m}_{\mathrm{e}} \cdot \mathrm{c}^{2} \cdot \mathrm{h}^{2}}}$

In this equation, assuming that the temperature $T$ is the body temperature $\left(T_{c}\right)$ and $V_{0}$ is the appropriate voltage for water composition $\left(\mathrm{V}_{\mathrm{H}_{2} \mathrm{O}}\right)$, the final equation (15) is formed.

$\Delta \mathrm{f}=\sqrt{\frac{6 \cdot \mathrm{k}_{\mathrm{B}} \cdot \mathrm{T}_{\mathrm{C}} \cdot \mathrm{e}^{2} \cdot \mathrm{V}_{\mathrm{H}_{2} \mathrm{O}}^{2}}{\mathrm{~m}_{\mathrm{e}} \cdot \mathrm{c}^{2} \cdot \mathrm{h}^{2}}}$

Equation (15) consists of constants, indicating that variation in frequency, in order to produce $\mathrm{H}_{2} \mathrm{O}$ and $\mathrm{ATP}$, is very well defined. The permissible frequency values are not random. The same is already shown for natural frequency $\mathrm{f}_{0}$ - equation (6).

\subsection{Calculations}

Table 1: Constants used (S. I. units)

\begin{tabular}{|l|l|}
\hline Speed of light in vacuum & $2.997924580 \cdot 10^{8}$ \\
\hline Electron mass & $9.109383560 \cdot 10^{-31}$ \\
\hline Planck's constant & $6.626070041 \cdot 10^{-34}$ \\
\hline Electron / elementary charge & $1.602176565 \cdot 10^{-19}$ \\
\hline Body temperature & $3.097500000 \cdot 10^{2}$ \\
\hline Water voltage & $1.230000000 \cdot 10^{0}$ \\
\hline Boltzmann's constant & $1.380648520 \cdot 10^{-23}$ \\
\hline
\end{tabular}

Applying values of TABLE 1 in equations (6), (15) and (12), values of TABLE 2 are obtained.

Table 2: Calculated values for natural frequency, variation, percentage of variation and mean velocity (S. I. units)

\begin{tabular}{|l|l|l|l|}
\hline $\mathrm{f}_{0}$ & $\Delta \mathrm{f}$ & $\frac{\Delta \mathrm{f}}{\mathrm{f}_{0}} \cdot 100 \%$ & $v$ \\
\hline $5.948 \cdot 10^{14}$ & $1.665 \cdot 10^{11}$ & $0.02799 \%$ & $8.392 \cdot 10^{4}$ \\
\hline
\end{tabular}

Applying values of TABLE 2 in (16) and (17):

$$
\begin{aligned}
& \mathrm{f}_{\text {max }}=\mathrm{f}_{0}+\frac{\Delta \mathrm{f}}{2} \\
& \mathrm{f}_{\text {min }}=\mathrm{f}_{0}-\frac{\Delta \mathrm{f}}{2}
\end{aligned}
$$

the upper and lower limits of frequency are determined. TABLE 3 shows their values:

Table 3: Minimum, optimal and maximum frequency (S. I. units)

\begin{tabular}{|l|l|l|}
\hline$f_{\min }$ & $f_{0}$ & $f_{\max }$ \\
\hline $5.947 \cdot 10^{14}$ & $5.948 \cdot 10^{14}$ & $5.949 \cdot 10^{14}$ \\
\hline
\end{tabular}




\section{Discussion}

(In part 4, the previously described model is correlated to calculations. Comments are numbered and refer to calculated values or relations from part 3. Summary is also provided.)

1) In order to gather at the same place all reactants, it is necessary for the system to have enough energy, so as to produce water successfully. A process so crucial for mitochondria, such as the process to produce ATP, should be of high accuracy. This could explain why the value of $\Delta \mathrm{f}$ is small, calculated as percentage of $\mathrm{f}_{0}$ - TABLE 2.

2) Body temperature is a suitable biological variable to affect the quantized aerobic metabolism. Temperature affects the frequency variation $\Delta f(15)$ and kinetic energy of $\mathrm{CP}(7) . \mathrm{V}_{\mathrm{H}_{2} \mathrm{O}}$ affects the natural frequency value $\mathrm{f}_{0}(6)$ and the frequency variation $\Delta \mathrm{f}(15)$.

3) If kinetic energy of $\mathrm{CP}$ is higher than the required value for water production, may lead to breaking down the potential barrier, but CP may not participate in the reaction (CPs are not entirely captured by -O-O-). It is therefore possible to grab one of the two electrons of one of the pairs, so oxygen is released again but in another form, other than the expected molecular oxygen $\mathrm{O}_{2}$. Capturing one electron less creates ROS.

Summarizing suggested model's operation, it is possible to distinguish three possible ways, with respect to CP's kinetic energy:

1) When frequency exceeds $f_{\max }$, kinetic energy of $\mathrm{CP}$ is much more than appropriate to produce water. On one hand this results in breaking the bonds of $\mathrm{ON}$ state $\left(\mathrm{Fe}^{+3}-\mathrm{O}-\mathrm{O}-\mathrm{Cu}^{+1}\right)$ but, on the other, a single electron is captured, not $\mathrm{CP}$ in total. In this case the system switches to OFF state $\left(\mathrm{Fe}^{+2}-\mathrm{ROS}-\mathrm{Cu}^{+2}\right)$ and $\mathrm{Fe}^{+3}$ as well as $\mathrm{Cu}^{+1}$ change oxidation state, with the exact same way they would do in the successful production of water, only now there is no water but ROS instead. This also means, observation of $\mathrm{Fe}^{+2}$ alone cannot lead to a conclusion about whether the system is functioning properly or not.

2) When frequency is between $f_{\min }$ and $f_{\text {max }}, C P$ has adequate kinetic energy and can cleave bonds in such a way that lead to water and further to ATP production. The $\mathrm{Fe}^{+3}$ and $\mathrm{Cu}^{+1}$ change oxidation states, as in the first case above, but now water is produced successfully, avoiding ROS formation.

3) When frequency is below $f_{\min }, \mathrm{CP}$ does not have the appropriate kinetic energy (voltage is not high enough). Therefore, $\mathrm{CP}$ cannot break the barrier of $\mathrm{ON}$ state $\left(\mathrm{Fe}^{+3}-\mathrm{O}-\mathrm{O}-\mathrm{Cu}^{+1}\right)$, is repelled to $\mathrm{Cu}^{+1}$ by $-\mathrm{O}-\mathrm{O}-$, semiconductor remains in $\mathrm{ON}$ state and iron remains in the active form $\mathrm{Fe}^{+3}$. But the amount of $\mathrm{Fe}^{+3}$ will eventually increase because carriers will keep accumulating it. This will lead to voltage increase and incoming $\mathrm{CP}$ will acquire more kinetic energy, then the previous ones. Therefore, optimal function can be recovered when frequency is below $f_{\min }$, by natural mitochondrial operations. The same it cannot be said when frequency exceeds $f_{\text {max }}$.

So the amount of $\mathrm{Fe}^{+3}$ must be within limits as well. Too much $\mathrm{Fe}^{+3}$ leads to ROS, too little cannot set the oxygen free.

Table 4: Summary of predicted change of concentration for $\mathrm{Fe}^{+3}, \mathrm{Fe}^{+2}, \mathrm{H}_{2} \mathrm{O}$, ATP and ROS

\begin{tabular}{|c|c|c|c|c|c|}
\hline & $\mathrm{Fe}^{+3}$ & $\mathrm{Fe}^{+2}$ & $\mathrm{H}_{2} \mathrm{O}$ & ATP & $\mathrm{ROS}$ \\
\hline $\mathrm{f}<\mathrm{f}_{\min }$ & $\uparrow$ & $\downarrow$ & $\square \downarrow$ & $\downarrow$ & - \\
\hline $\mathrm{f}_{\min } \leq \mathrm{f} \leq \mathrm{f}_{\max }$ & $\downarrow$ & $\uparrow$ & $\uparrow$ & $\uparrow$ & - \\
\hline $\mathrm{f}>\mathrm{f}_{\max }$ & $\downarrow$ & $\uparrow$ & $\downarrow$ & $\downarrow$ & $\square \uparrow$ \\
\hline
\end{tabular}

Table 5: p-n-p states in relation to frequency values

\begin{tabular}{|l|l|l|}
\hline & State: ON & State: OFF \\
\hline $\mathrm{f}<\mathrm{f}_{\text {min }}$ & $\mathrm{Fe}^{+3}-\mathrm{O}-\mathrm{O}-\mathrm{Cu}^{+1}$ & $\begin{array}{l}\text { CP Kinetic Energy not enough to break the voltage } \\
\text { barrier. It can't go OFF! }\end{array}$ \\
\hline $\mathrm{f}_{\text {min }} \leq \mathrm{f} \leq \mathrm{f}_{\max }$ & $\mathrm{Fe}^{+3}-\mathrm{O}-\mathrm{O}-\mathrm{Cu}^{+1}$ & $\mathrm{Fe}^{+2}-\mathrm{O}_{2}-\mathrm{Cu}^{+2}$ \\
\hline $\mathrm{f}>\mathrm{f}_{\max }$ & $\mathrm{Fe}^{+3}-\mathrm{O}-\mathrm{O}-\mathrm{Cu}^{+1}$ & $\mathrm{Fe}^{+2}-\mathrm{ROS}-\mathrm{Cu}^{+2}$ \\
\hline
\end{tabular}

TABLE 4 presents this summary with arrows. To better understand the way this table is used, an example follows: Let's consider the case that the system operates in higher than $f_{\max }$ frequency. The model predicts that concentration of $\mathrm{Fe}^{+3}$ will decrease during $\mathrm{CP}$ 's operation (because in this case $\mathrm{Fe}^{+3}$ is converted to $\mathrm{Fe}^{+2}$ ), so the first arrow, corresponding to $\mathrm{Fe}^{+3}$, points down. For the same reason concentration of $\mathrm{Fe}^{+2}$ will 
increase - arrow points up. Water and ATP are not produced because no oxygen is produced, so they decrease. ROS concentration will be found higher as well. From TABLE 4 it is also clear that checking $\mathrm{Fe}^{+3}$ and $\mathrm{Fe}^{+2}$ concentrations alone, situations where $\mathrm{f}_{\min } \leq \mathrm{f} \leq \mathrm{f}_{\max }$ and $\mathrm{f}>\mathrm{f}_{\max }$ cannot be distinguished. This is also visible in TABLE 5, where the p-n-p state is related to frequency.

\section{Conclusions}

In order to help readers to remember them, conclusions are presented as Q \& A while the model is represented as a diagram (Fig.7)

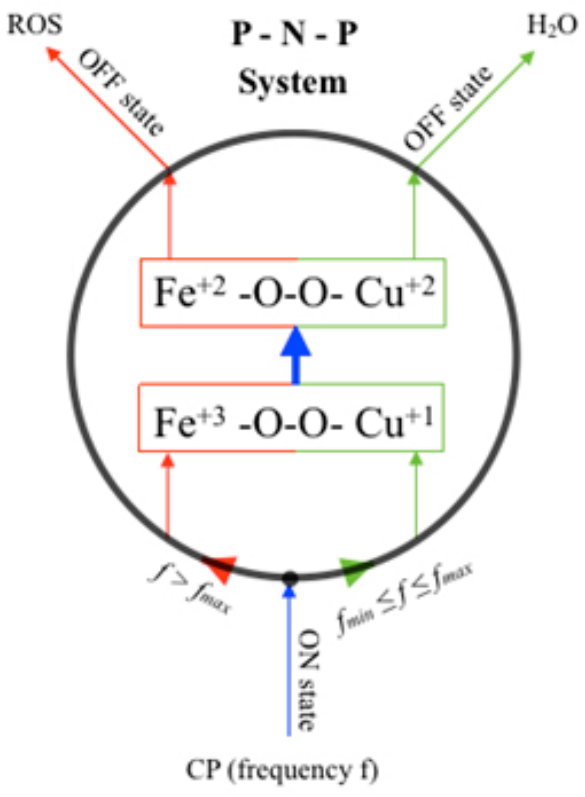

Figure 7: Diagram that describes the proposed model

\section{Q: What is the role of electrons in water production inside mitochondria?}

A: Electrons are used to break the bonds between metals and oxygen and release the oxygen. The mechanism described is a model for redox reaction inside mitochondria, resulting to oxygen release. Electrons also participate in the reaction of water synthesis, after oxygen release. However not every incoming electron pair leads to water formation.

2. Q: Assuming an electron pair activates the water production mechanism. Is it possible to produce both water and ROS?

A: Water production and ROS production in mitochondria are possible outcomes of the same mechanism but are not produced simultaneously. For every electron pair, the result it will be either water or ROS, not both.

3. Q: Assuming an electron pair activates the water production mechanism. What is the role of CP's frequency in the possible outcome?

A: CP's frequency is very important to this mechanism, as already shown, because it affects CP's kinetic energy, which furthermore affects CP's capability to free oxygen in the correct form. There is a narrow band of frequencies ( $\Delta \mathrm{f})$ that results to water. Frequencies above a certain value $\left(\mathrm{f}_{\max }\right)$ produce ROS.

4. Q: Assuming an electron pair activates the water production mechanism. How CP's frequency value is determined?

A: Whatever affects voltage between $\mathrm{Fe}+3$ and $\mathrm{Cu}+1$ determines $\mathrm{CP}$ 's frequency. However the easier to change to make mitochondria dysfunctional leading to ROS production, is the value of $\mathrm{Fe}^{+3}$ concentration. Increase it and it will increase CP's kinetic energy, therefore $\mathrm{Fe}^{+3}$ concentration can be considered the main factor to determine CP's frequency.

\section{Q: How this mechanism can be used to prevent ROS production?}

A: TABLE 5 can provide a guide to set the boundaries between the three states of mitochondrial function, by measuring concentrations of $\mathrm{Fe}^{+3}, \mathrm{Fe}^{+2}, \mathrm{H}_{2} \mathrm{O}$, and $\mathrm{ROS}$. The way these values change, can point at the frequency range that paired electrons operate. Then, adjusting $\mathrm{Fe}^{+3}$ concentration, the system can change its behavior accordingly. Adjusting $\mathrm{Fe}^{+3}$ concentration is one way to try to regulate ROS production experimentally. Because moving electrons form current, application of appropriately oriented external electric and magnetic fields can also affect this procedure, favoring ROS or water production. 
So, if the hypothetical model is valid, ROS production can be regulated (even prevented) by adjusting $\mathrm{Fe}^{+3}$ concentration or by any other way that affects CP's frequency.

\section{Q: What is the contribution of this work to science?}

A: Valid or invalid, this model provides a solid ground to start examining this crucial mitochondrial procedure that produces either water and ATP or ROS. It is not just an interesting procedure to examine, but also critical for mitochondrial health, which extends to mammalian health, therefore is worthy of attention. More to this, if this model is valid, it presents a clear - fresh - look in a mitochondrial procedure that is completely unknown until now. So, if this hypothesis is valid, reviewing only Fig.7, equations (6), (15), (16) (17) and TABLES 4, 5 one would know what affects ROS production, where to look to change system's behavior and how to use this model to improve mitochondrial health.

\section{References}

[1]. J. Bardeen, L. N. Cooper, and J. R. Schrieffer, Theory of superconductivity, Physical Review, 108(5), 1957, 1175-1204.

[2]. A. A. Abrikosov, The magnetic properties of superconducting alloys, Journal of Physics and Chemistry of Solids, 2(3), 1961, 43-46.

[3]. B. S. Deaver and W. M. Fairbank, Experimental evidence for Quantized flux in Superconducting cylinders, Physical Review Letters, 7(2), 1961, 43-46.

[4]. B. D. Josephson, Possible new effects in superconductive tunneling, Physics Letters, 1(7), 1962, 251-253.

[5]. G. M. Cooper, The cell: A molecular approach, 2nd ed. (Washington, D.C., ASM Press: Sinauer Associates Inc., 2000).

[6]. B. Alberts et al., Molecular biology of the cell, 4th ed. (New York, Garland Publishing, 2007).

[7]. M. Drewsen and A. Brøner, Harmonic linear Paul trap: Stability diagram and effective potentials, Physical Review A, 62(4), 2000. 